\title{
Effects of guided inquiry virtual and physical laboratories on conceptual understanding, inquiry performance, scientific inquiry self-efficacy, and enjoyment
}

\author{
Siti Jamiatul Husnaini ${ }^{1}$ and Sufen Chen ${ }^{1,2, *}$ \\ ${ }^{1}$ Graduate Institute of Digital Learning and Education, \\ National Taiwan University of Science and Technology, Taipei 10607, Taiwan \\ ${ }^{2}$ Optentia Research Focus Area, North-West University, Vanderbijlpark 1900, South Africa
}

(Received 31 May 2018; published 25 March 2019)

\begin{abstract}
Indonesia and many other developing countries have a vast youth population, yet limited facilities for physics learning. The major purposes of this study are to develop low-cost, technology-enhanced physical and virtual laboratories and to investigate their effects on various learning objectives, including conceptual understanding, inquiry performance, scientific inquiry self-efficacy, and enjoyment. The virtual laboratory (VL) used the physics education technology to simulate a pendulum, while the physical laboratory (PL) was a technology-enhanced physical laboratory utilizing the Camera Stopwatch and Smart Tools applications. In this quasiexperimental design, a total of 68 secondary school students in Indonesia were randomly assigned to the PL and VL settings. The participants conducted the pendulum experiment guided by an inquiry worksheet along with pre- and postconceptual tests, scientific inquiry self-efficacy, and enjoyment questionnaires. The result revealed that the guided inquiry-based VL was as effective as the PL for simple concepts, but was more effective for improving difficult concepts and scientific inquiry selfefficacy. Nevertheless, the PL group performed better on crucial inquiry activities, that is, planning, experimenting, and further improvement of the experiment. Moreover, both the PL and VL significantly promoted enjoyment. It was concluded that the PL and VL were successful for achieving different learning objectives.
\end{abstract}

DOI: 10.1103/PhysRevPhysEducRes.15.010119

\section{INTRODUCTION}

The use of technology-enhanced laboratories to improve inquiry is an evolving issue in many countries. Their effects have been evinced in numerous studies by applying various technologies such as simulation [1-4], microcomputerbased laboratories [5-8], remote laboratories [9,10], and augmented reality $[11,12]$. The use of technology can support students' experience of inquiry and the constructive process of science in the laboratory [13]. Moreover, the technology could make inquiry less demanding for students by eliminating unrelated factors [13]. In the laboratory activities, students may be discouraged when they fail to get the expected results after following procedures and not being able to figure out what was wrong [14] because many traditional instruments are unreliable. For example, heat and temperature experiments cannot avoid environmental influences on the calorimeter. This could increase students'

*sufchen@mail.ntust.edu.tw

Published by the American Physical Society under the terms of the Creative Commons Attribution 4.0 International license. Further distribution of this work must maintain attribution to the author(s) and the published article's title, journal citation, and DOI. cognitive load and restrict their ability to draw meaningful conclusions. Technology-enhanced laboratories increase the number of successful experiences, which are important for cultivating competence [13]. In addition, technologyenhanced laboratories could reduce the time needed for inquiry. Time constraints are a major reason why technology should be used to replace traditional laboratories [15]. Simulation could save the time of data collection for analysis, discussion, and evaluation.

Technology use for inquiry has grown considerably in developed countries (see Table I), but it has not yet spread widely to assist 21 st-century students in developing countries (e.g., Indonesia). Nor have many studies on technology-based learning been conducted in Indonesia [16]. This situation could be caused by both the fact that most teachers do not have the means or capability to use technology in promoting learning [17] and the lack of technical support [18-20]. Indonesia encounters particular challenges due to poor network systems and shortages of ICT equipment, inadequate government policy and uncoordinated administration systems, lack of teachers' and learners' knowledge, and lack of teachers' motivation [16]. Quality research yields practical solutions to some of these problems and inputs momentum for innovative teaching. This study thus aimed to develop and evaluate affordable technology-enhanced laboratories for Indonesian 


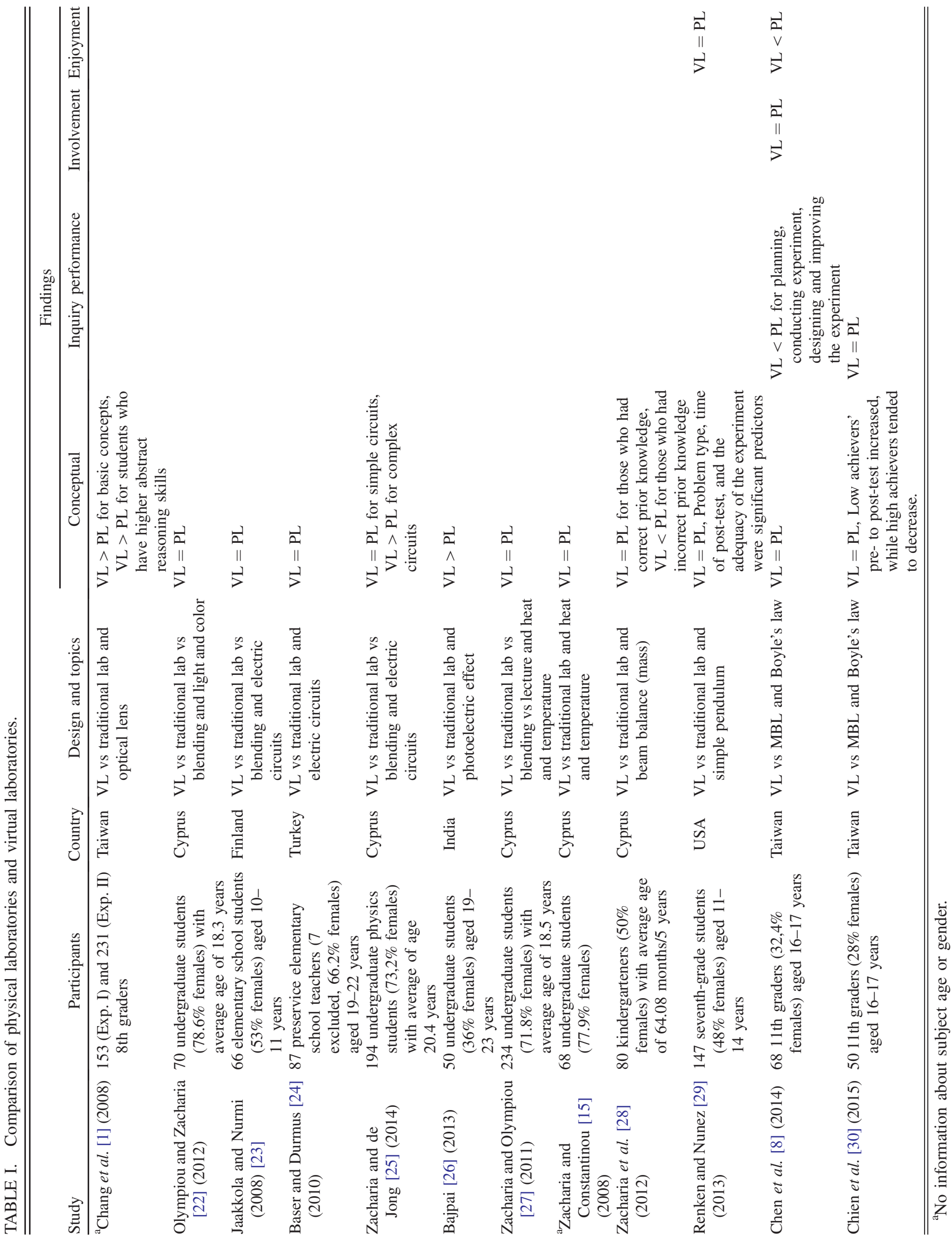


secondary school students. The results could help Indonesian teachers, as well as teachers from other developing countries, to understand students' inquiry learning, and to select and design appropriate laboratories for their students.

Table I lists a summary of the experimental studies in the last 10 years (2008-2018) involving technology-enhanced laboratories for inquiry in physics. The review focused on physics, inquiry, and comparisons between virtual and physical laboratories or with lectures. Based on Table I, it was found that most studies were done in countries where the curricula were strongly influenced by western countries. The early Indonesian educational system had some input from the Dutch, but overall it has experienced autonomous development. It has a strong cultural background where students respect and listen to, but do not challenge or question teachers; thus, teaching is highly teacher centered [21]. Marambe et al. [21] surveyed university freshmen in Indonesia, Sri Lanka, and Netherlands. Indonesian students reported relatively frequent use of memorizing and rehearsing strategies compared with Sri Lankan and Dutch students. Their conceptions of learning were more diploma, self-test, and vocation oriented, and they viewed learning as intake and use of knowledge, rather than inspiration or collaboration. Inquiry learning, which emphasizes deep processing, critical thinking, the use of metacognitive strategies, and active learning, appears to be challenging to educational systems such as this. This study aimed to fill this research gap by investigating the learning effects of a guided inquiry-based physical laboratory (PL) and virtual laboratory (VL) in Indonesia.

\section{A. Inquiry}

This current study defines inquiry as various activities in which scientists explore and interpret the world based on evidence [31]. Inquiry learning refers to both an instructional approach and curriculum materials for the students to learn science and scientific ways of obtaining knowledge [32-34]. Based on the extent to which a laboratory investigation provides guidance, the inquiry could be categorized as confirmation, structured inquiry, guided inquiry, open inquiry, or authentic inquiry [35]. This current study was designed as a guided inquiry to enable students who lacked experience in laboratories to conduct experiments without teacher instruction, which could become teacher centered according to the culture, thus eliminating the essence of inquiry. The laboratory manual provides the problem, the background, and guidance of the procedures, but the methods of analysis, interpretation, and conclusion are for the students to generate [35]. For example, the guided-inquiry worksheet provided information about the problem to test the relationship between the period and the length of the pendulum, but asked students to select the learning objectives and establish a plan before the experiment, such as the amount of data to collect and operational variables. Moreover, the review section presented eight questions to guide the students to reason the possible relationships between variables. Guided inquiry, rather than open or authentic inquiry, was chosen for a number of reasons. First, the subjects in the current study were considered to be low achievers based on their past academic performance. Second, they had not previously experienced experiment settings. Overall, Indonesia still lacks laboratory activities to support learning processes [36] because the ability of teachers to design inquiry laboratory activities is still low [37] and the facilities are not adequate [16]. Consequently, most teachers teach students by lecturing, and seldom adopt inquiry-based approaches. The few laboratories students had, if any, were at the level of confirmation and were structured.

\section{B. Theoretical framework and empirical studies on PL and VL}

The technology-enhanced laboratories involved in this study were a pendulum VL using the physics education technology (PhET) and a PL utilizing the Camera Stopwatch and Smart Tools applications. Bivall, Ainsworth, and Tibell [38] stated that learning is strongly influenced by sensory-motor processing. According to embodied cognition [39,40], human's neural resources are devoted principally to perceptual and motoric processing whose cognitive activities consist of direct interaction with the environment. As a result, learning with multiple inputs from the environment such as visual, tactile, auditory, and linguistic inputs may result in better outcomes. Compared with mainly visual input in the VL settings, the embodied cognitive theory supports PL. On the other hand, cognitive loading theory offers a different view for multiple inputs. Human working memory can handle only a limited amount of information [41]. Multiple inputs and interactions may cause high cognitive load [42]. The inherent features and design of VL could resolve the students' intrinsic cognitive load.

If a VL creates learning effects as well as physical laboratories do, educators and policy makers would opt for the VL because of its apparent extra advantages such as low cost per use (assuming that computers, the Internet, or smartphones are available), its convenience, manageability, and affordance of multiple representations. Thus far the comparison between PL and VL demonstrates inconsistent results regarding whether VL are more effective than or equally as effective as PL (Table I). Some researchers have found that students' conceptual understanding in virtual laboratories outperformed that of students in physical laboratories $[1,26]$. Other researchers have found that the effects are similar in terms of students' achievement $[8,15,22,24,29]$. Chen et al. [8] argued that more specific experiments should be carried out to more clearly understand the effectiveness of VL. First of all, the information 
accessibility and dynamic visualization provided by VL and PL are not equal. For example, in the laboratories about dynamic electricity, VL students could observe the current and electron flows inside a bulb on the screen, something which is absent from a PL $[2,25,43]$. Hence, PL students may encounter process-related problems involving averted development of an appropriate conceptual model because only the VL affords a view of the current flow [25]. Moreover, the post-laboratory test may favor the VL group because of using similar representations. If the PL group had access to the same amount of information, the comparison results might have been different.

Second, students' prior knowledge or experience might supplement their sensory learning in a physical laboratory. Consequently, physicality is not necessary for students to obtain a higher score if the concept could be constructed without further sensory-motor information or if students have previous experience of manipulating the objects. However, for many science concepts, drawing from the research in neuroscience $[44,45]$, motor experience may still play a significant role in the learning.

Third, the effectiveness of PL and VL in terms of integrated cognitive and affective learning objectives is missing. Most previous studies focused on and emphasized conceptual learning (Table I), while laboratory learning could pursue a whole range of learning objectives (e.g., cognitive, affective, and motor) to generate more convincing results $[13,46,47]$. Indeed, an extensive review of papers on the comparison studies of nontraditional (virtual and remote) versus traditional (hands-on) laboratories [48] found that the inquiry performance was the least assessed learning objective $(n=4,7 \%)$. Inquiry performance assesses how students perform the inquiry task, where students make observations, ask questions, develop and use a model, and plan and carry out an investigation [49]. Similarly, scientific inquiry self-efficacy (SISE) has not received adequate attention, either. Self-efficacy is a student's belief in their ability to perform specific tasks or processes and to successfully achieve designated results [50-52]. As for inquiry, it can be defined as a student's perceived capability to perform the tasks corresponding to scientific inquiry [53]. Students with high SISE are more likely to work harder at an inquiry task, to see the difficulty as a challenge [53], and to successfully achieve learning goals [54] over less self-efficacious students. Self-efficacy has been reported as a strong predictor of academic achievement [54], and inquiry-based curricula could act as a catalyst to improve students' self-efficacy $[53,55]$. As a result, SISE was considered as an important dependent variable in the current study. Finally, inconsistent results of affective aspects, that is enjoyment, were found from previous studies. Students mentioned that PL was more enjoyable than VL in postlaboratory interviews [8], Rice et al. [56] and Rong et al. [57] found that VL enhanced the enjoyment of learning, while Renken and Nunez [29] reported that PL is as enjoyable as VL. Therefore, when evaluating laboratories for Indonesian secondary schools, the enjoyment factor was taken into consideration. Considering the objectives of the laboratories according to the literature and the National Science Education Standards [52], a systematic and thorough evaluation of these two technology-enabled lab formats would cover the above-mentioned variables to understand and enhance their values.

\section{Research question}

This study was driven by the following research question:

To what extent do guided inquiry-based PL and VL affect conceptual understanding, inquiry performance (IP), scientific inquiry self-efficacy, and enjoyment?

\section{THE STUDY}

This study aimed to investigate the effectiveness of the VL and PL on students' conceptual understanding, IP, SISE, and enjoyment using the same learning method, a guided inquiry activity. Typically, a physical laboratory enables students to manipulate real tools, while virtual laboratory activities allow students to manipulate virtual materials on a PC screen or mobile device. Because of the wide adoption of technology-enhanced laboratories, there are some physical laboratories utilizing technology in which students still manipulate real tools but merged with technology, such as microcomputers from PASCO and using apps to avoid disproportionate information accessibility, technology affordance, and measurement quality. The PL in this study was not a traditional laboratory. It used a technology-enhanced simple pendulum. Applications (apps) such as Stopwatch Camera were used to count the period, and Smart Tools were used to measure the length of the pendulum rope and deviation angle. Moreover, most available VLs outside mechanics come with dynamic visualization and multiple representations such as molecular movement, which may affect the students' understanding. In order to control these factors, we decided to use topics in mechanics, out of which pendulums had not been conducted in the participants' middle schools. Hence, pendulum motion was selected so that the results would not be disturbed by previous physical manipulation. What students looked at and manipulated on the PC screen in the VL class was similar to what the students did in the PL.

This was a comparison study of pre- to post-tests in the control and experimental groups. Both groups were provided with the same guided inquiry-based worksheet including nine sections. In each section, guiding questions were presented to promote learning. The summary of students' activities and examples of the guiding questions are presented as follows:

(1) Goal setting. Students set their own learning goals for the laboratory. They were asked: What learning 
objective (s) would you like to achieve in this laboratory? A list of six objectives such as to design an experiment to answer a question and describe the relationship between the period $(T)$ and the length of pendulum rope $(L)$ were provided for students' reference.

(2) Review of the concept. Students reviewed the pendulum motion concept by responding to eight questions presented as paragraphs. For example, they were guided to think about their experience on a swing: if you want to go faster, which means $T$ is $\square$ smaller or $\square$ bigger, the rope should be $\square$ longer or $\square$ shorter. They clicked on the boxes. After several similar guiding questions, they tried to formulate the simple pendulum equation.

(3) Constructing an experiment question and identifying variables. Students constructed the experiment question to investigate the relationship between $T$ and $L$ and identified the variables. An example of the latter was as follows: Based on your research question, what are the control, independent, and dependent variables?

(4) Planning. The students made several important decisions about the experiment design. For example, they had to think about how many data points should be collected to convince others that the result was trustworthy and why, and the initial deviation of the pendulum.

(5) Experimenting. The students conducted experiments and recorded data. They had to decide which variables to record and had to calculate the gravitational acceleration.

(6) Evaluating, designing, and improving the experiment. The students evaluated the possible experimental flaws, designed an improvement, and then improved the experiment. They were guided to observe their own results as well as those of the other groups to evaluate if the equation $\mathrm{T}=$ $2 \pi \sqrt{L / g}$ is applicable to all situations. Then they were encouraged to use text/figures to describe their further improvement of the experiment.

(7) Analyzing errors and plotting graphs. The students analyzed and observed whether the errors increased or decreased from the first to the second experiments, followed by plotting the $L-T$ and $L-T^{2}$ graphs based on their own data.

(8) Interpreting results. The students interpreted their results. For example, they explained the meaning of the slope.

(9) Designing a new experiment. Each student proposed a new research question and designed a new experiment to answer the question.

This framework was modified by following the suggestion of Chen et al. [8] and Chien et al. [30], where they moved the design of a completely new experiment to the last part of the worksheet and reduced the guidance in the second half of the worksheet.

During nine tasks, the students conducted the experiment twice, in sections V and VI. They conducted the second experiment after analyzing the experimental flaws in the first experiment. Most students experimented with the pendulum using a big deviation (e.g., $>10^{0}$ ); however, the pendulum formula worked only on a small deviation. The smaller the deviation, the smaller error because of the small deviation. Both groups took the same $135 \mathrm{~min}$ to conduct the experiment and fill in the worksheet. Each of the pre- and post-tests and questionnaires took $45 \mathrm{~min}$ to complete.

\section{METHODOLOGY}

\section{A. Sample}

The participants of this study were 68 10th graders (54 females, 14 males; $M_{\text {age }}=15.8$ years, $\mathrm{SD}=0.67$ ) of a public secondary school in Bangkalan, Indonesia. The school taught religious affairs $10 \mathrm{~h}$ per week in addition to regular subjects. Based on the students' past performance on the entrance exam and taken into account the context of their educational background and opportunities so far in life, they were considered as low achievers. Compared with high achievers, low achievers benefit more from guidance in inquiry-based experiments [30]. Therefore, we chose this group of students for this study. The participants were randomly assigned to a control group (PL) and an experimental group (VL), each consisting of 34 students.

\section{B. Curriculum materials}

This study centered on the simple pendulum module of the guided inquiry curriculum. The goals were for the students to set experiment objectives, review the concepts, plan, conduct an experiment, analyze the experimental flows, improve an experiment, plot the graphs, interpret data, and design a new experiment. One week before the laboratories, the teacher trained all participants in how to use the apps. Then the teacher gave brief explanations of the above-mentioned goals at the beginning of the session. The instructor informed the students that they would conduct the experiment twice. If the gravitational acceleration was not $9.8 \mathrm{~m} / \mathrm{s}^{2}$ in the first experiment, they needed to find the variables that affected it, and try to fix it in the second experiment. Since instructors' intervention significantly influences students' learning in a laboratory [58] and an inquiry [59], there was no teacher instruction during the laboratories so as to avoid unequal interventions and confounded results due to teacher instruction. Students were randomly assigned by the teacher to work in pairs, and each submitted individual reports for grading.

\section{Manipulatives}

In the traditional pendulum laboratory, students are usually asked to count the pendulum time for 10 harmonic 
motions using a stopwatch, then divide it by 10 to get the period along with measuring the deviation angle using a protractor. There will be some errors due to the air friction, human error, and parallax. Human errors are usually caused by switching on and off the stopwatch, and parallax errors are due to deviation angles often being ignored or not measured. In the current study, the PL students manipulated objects by engaging real equipment (e.g., stative rods, bobs, ropes) with technology support to measure the period and deviation. They utilized apps, such as Stopwatch Camera and Smart Tools, to root the physical laboratory in a more precise way. How Stopwatch Camera works is based on dividing the recorded video into frames so users can choose a range of what frame number they want to determine the time. The Smart Tools app provides some measurement functions including a ruler and protractor. Students could exploit the protractor to measure the deviation angle. These apps could work with a camera mobile phone and the data shown on the screen. On the other hand, the VL students manipulated the virtual instruments in the PhET. Experiments are set up by ticking the photogate timer, determining the mass and length, dragging the bob to a certain deviation, and clicking the start icon on the photogate timer.

\section{Measures}

A conceptual test was administered to assess the students' understanding of pendulum-related concepts. In the current study, four pendulum concepts were covered in 10 items:

(1) Simple harmonic motion.

(2) Simple pendulum formula and application, that is, how much the percentage of the pendulum length should be changed if gravitational acceleration increases while the period remains the same, and calculating the pendulum length while the other variables are provided (e.g., the amount of swing in $1 \mathrm{~min}$ )

(3) Analyzing data and $L-T$ graphs.

(4) Creating hypotheses and variables.

Based on the conceptual pretest, the test items were split into simple and difficult questions depending on the percentage of correctness. The multiple-choice questions were collected from several previous unpublished Indonesian studies with high internal consistency in the range of $0.75-0.91$ and the content validity based on experts' reviews. The test was piloted with 60 high school students and the $K R 20$ was 0.74 . Moreover, students' IP was measured by analyzing their responses on the worksheet. However, we noted that this metric might not be an accurate measure of IP, since we would show that many VL students did not pay much attention to the worksheet; see Sec. VII. SISE and enjoyment data were collected by questionnaires which were coded using a 5-point Likert scale ranging from strongly disagree $(=1)$ to strongly agree
$(=5)$. The SISE questionnaire was adopted from Ketelhut's [60] Scientific Inquiry Self-Efficacy. Reliability $(\alpha=0.90)$ and content and construct validity were established in the original paper. Sample items include the following: "I can use graphs to show what I found out in my experiment," "It is hard for me to look at the results of an experiment and tell what they mean," and "I can design an experiment to test my ideas." The enjoyment questionnaire was developed by Liang et al. [61], and was found to have acceptable factorial and criterion validity, internal consistency $(\alpha=0.93)$, and test-retest reliability. The seven items were framed in the context of "When I am doing lab activities..." “...I feel bored" or "...I dislike it." They were expressed negatively, so higher scores indicated lower enjoyment. The ratings were reversed for analysis.

The worksheet and questionnaires were translated from English into Indonesian by the researcher, instructor, and one Master's student. The instruments were piloted with three students to ascertain that they understood the meaning of both the worksheet and questionnaire statements as the researchers expected. Based on the pretest data, the construct validity was examined by confirmatory factor analysis. For SISE, correlations were found between two items and four out of 12 items were deleted due to their low factor loadings. For enjoyment, two correlations between items 1 and 2 and between items 4 and 5 were identified in our model. The analyses demonstrated good model fit for both SISE and enjoyment. CFI and TLI were above the criterion of 0.95, SRMR was lower than the criterion of 0.09 [62], and the $\chi^{2} / \mathrm{df}$ ratio was lower than the recommended 2.0 [63]. SRMR, rather than RMSEA was used due to the small sample size [64].

\section{DATA ANALYSIS}

The analysis involved paired sample $t$ tests to compare the pre- to post-tests on simple and difficult concepts, SISE, and enjoyment, to investigate the effectiveness of each laboratory. ANCOVA was administered afterward to identify any differences between the PL and VL in the post-tests after the pretests were controlled as the covariates. Effect sizes, that is, Cohen's $d$, partial $\eta^{2}$, and their 95\% CI (CI 0.95 ), were estimated. The CI 0.95 was converted from the lower and upper limits of the CI 0.95 on the noncentrality parameter [65,66]. Moreover, independent $t$ tests were administered to compare the PL and VL goups' IP in the nine sections of the worksheet: setting goals, reviewing concepts, forming questions and identifying variables, planning, experimenting, further improvement of the experiment, analyzing errors and plotting graphs, interpreting data, and designing a new experiment.

Students' IP was graded by two teachers based on a rubric developed by the researchers. It has interrater reliability of 0.93 measured by Cohen's kappa. The total maximum and minimum scores were 69 and 0 . For questions that had correct answers, 0 and 1 were given to no response or 
TABLE II. Means, standard deviations, and comparisons of conceptual understanding in the pre- and post-tests.

\begin{tabular}{|c|c|c|c|c|c|c|}
\hline Group & Conceptual level & Pretest $M(\mathrm{SD})$ & Post-test $M$ (SD) & $t$ & $p$ & Cohen's $d^{\mathrm{a}}$ and Its CI 0.95 \\
\hline $\mathrm{PL}(n=34)$ & Difficult ${ }^{\mathrm{b}}$ & $4.71(7.06)$ & $6.76(5.89)$ & 1.42 & 0.165 & 0.31 CI $0.95=[-0.13,0.76]$ \\
\hline $\mathrm{VL}(n=34)$ & Difficult ${ }^{\mathrm{b}}$ & $7.65(8.90)$ & $11.47(10.19)$ & 1.63 & 0.113 & 0.40 CI $0.95=[-0.09,0.89]$ \\
\hline
\end{tabular}

${ }^{\mathrm{a}}$ Effect size $d=<0.3$ small effect, $0.5-0.8$ medium effect, and $>0.8$ large effect.

${ }^{\mathrm{b}}$ Highest possible score was 50 .

incorrect and correct answers. For open-ended questions, criteria based on relatedness, correctness, and rationality were created. For example, for data recording, the criteria were (i) data being taken systematically, (ii) correct calculation, and (iii) careful with the use of decimals. Then, 4, 3, 2 , and 1 were given when three, two, one, and none of the criteria were fulfilled, respectively, while 0 was assigned to no response. Similarly, the criteria for plotting graphs were correct shape of graphs, a systematic scale, and plotting based on their data. For analyzing experimental flaws, the criteria were (i) analyzing based on the data, (ii) logical reasoning, and (iii) using scientific concepts.

\section{RESULT}

\section{A. Conceptual understanding}

Based on paired $t$ tests, there was a statistically significant improvement from the pre- to post-tests in both the PL and VL for simple conceptual understanding, $t(33)=3.41$ and $5.26(p<0.01)$, respectively, and Cohen's $d=0.89$ and 1.21, denoting big effect sizes for both groups (Table II). The CI 0.95 of $d$ further revealed that the effect size of the PL ranged from medium to large, whereas the range of the VL was relatively larger.

Furthermore, there was no statistically significant progress after the PL and VL for difficult conceptual understanding, $t(33)=1.42$ and $1.63(p>0.05)$, respectively

\section{Simple Concept Score}

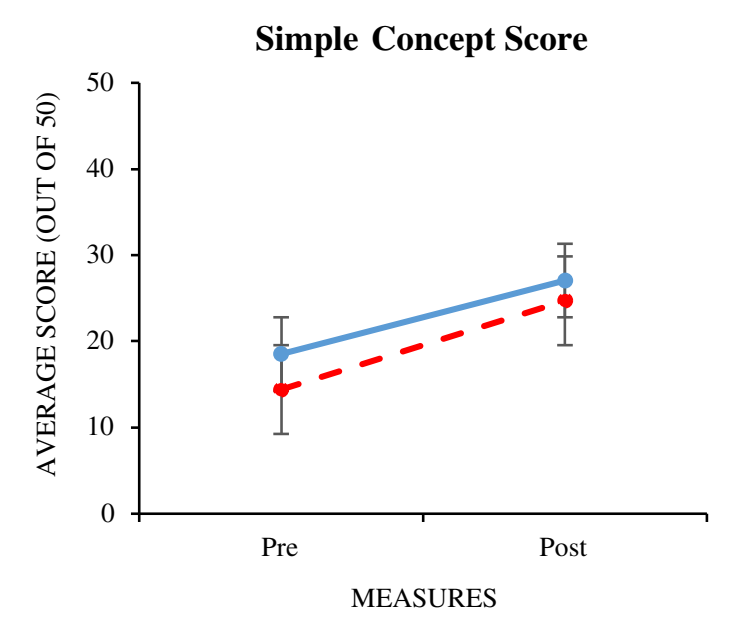

(a) (see Table II and Fig. 1). Moreover, ANCOVA found no significant difference for the simple concepts $(F[1,65]=$ $1.40, p=0.240$, partial $\left.\eta^{2}=0.021\right)$, but there was a significant difference for the difficult concepts $(F[1,65]=$ 4.98, $p=0.029$, partial $\eta^{2}=0.071$ ).

\section{B. Inquiry performance}

Independent $t$ tests were used to examine students' IP of nine sections starting with setting goals to design a new experiment, as shown in Table III.

It was found that the VL and PL students performed equally well in terms of analyzing errors and plotting graphs. This means that both groups had equal ability to figure out the experimental errors in different experiments, as well as scale graphs systematically and correctly. Moreover, the PL students performed significantly better than the VL students on several tasks, that is, selecting more learning objectives, reviewing the pendulum motion concept, constructing the experiment question and identifying variables, planning, experimenting and recording data, and evaluating, designing, and improving experiments. On the other hand, the VL group performed better than the PL group on the tasks of interpreting data and designing a new experiment. In sum, the effectiveness of the VL was different from that of the PL for students' IP. PL students performed better than VL students on several important inquiry activities, that is, planning,

\section{Difficult Concept Score}

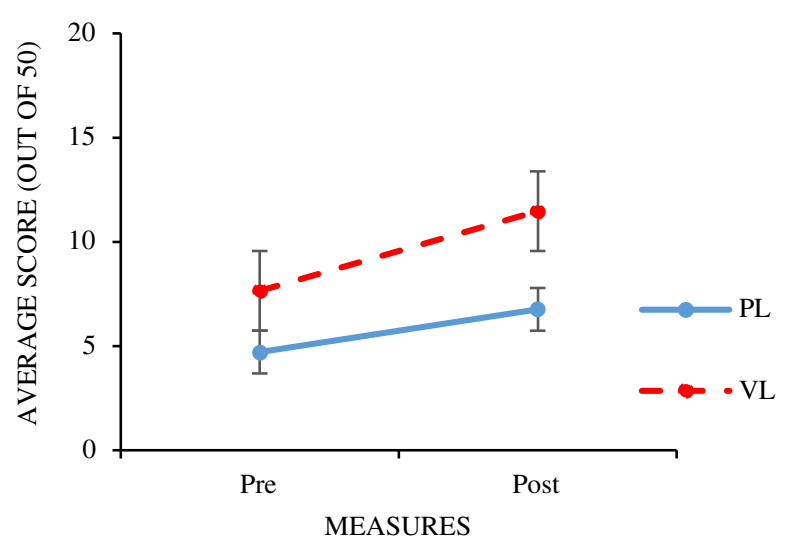

(b)

FIG. 1. (a) The enhancement of simple concepts for the PL and VL. (b) The enhancement of difficult concepts for the PL and VL. 
TABLE III. Comparison of students' IP.

\begin{tabular}{|c|c|c|c|c|c|c|}
\hline & $M(\mathrm{SD})^{\mathrm{b}}$ & $t$ & & & & \\
\hline Section & PL & VL & $d^{\mathrm{a}}$ and $\mathrm{CI} 0.95$ & Positive effect & PL & VL \\
\hline Setting goals & $4.50(2.30)$ & $1.97(0.97)$ & $5.91^{* *}$ & $1.43[0.89,1.96]$ & $\checkmark$ & \\
\hline Reviewing the pendulum motion concept & $5.68(1.53)$ & $4.62(1.60)$ & $2.79^{*}$ & $0.67[0.19,1.16]$ & $\checkmark$ & \\
\hline $\begin{array}{l}\text { Constructing the research question and } \\
\text { identifying variables }\end{array}$ & $3.56(.70)$ & $2.29(0.76)$ & $7.11^{* *}$ & $1.72[1.17,2.29]$ & $\checkmark$ & \\
\hline Planning & $8.71(1.53)$ & $6.35(2.27)$ & $5.02^{* *}$ & $1.22[0.70,1.74]$ & $\checkmark$ & \\
\hline Experimenting & $3.26(1.05)$ & $2.38(0.89)$ & $3.73^{* *}$ & $0.81[0.32,1.31]$ & $\checkmark$ & \\
\hline Evaluating, designing, and improving the experiment & $5.47(1.13)$ & $4.29(1.17)$ & $4.21^{* *}$ & $1.02[0.51,1.53]$ & $\checkmark$ & \\
\hline Analyzing errors and plotting graphs & $5.15(1.89)$ & $4.65(1.52)$ & 1.20 & $0.29[-0.19,0.77]$ & & \\
\hline Interpreting results & $2.65(1.01)$ & $3.29(0.97)$ & $2.69^{*}$ & $0.64[0.16,1.13]$ & & $\checkmark$ \\
\hline Designing a new experiment & $0.91(0.29)$ & $1.47(0.90)$ & $3.46^{*}$ & $0.84[0.34,1.34]$ & & $\checkmark$ \\
\hline
\end{tabular}

experimenting, and further improvement of the experiment. Finally, the VL group performed better on the last two sections of the worksheet which involved higher cognitive skills.

\section{Scientific inquiry self-efficacy}

Regarding SISE, the VL group improved significantly from the pre- to post-tests, $t(33)=2.81, p<0.01$, Cohen's $d=0.62$, CI $0.95=[0.15,1.10]$, but there was no significant difference in the PL group's result $[t(33)=1.96$, $p=0.059$, CI 0.95 $=[-0.01,0.66]]$ (Table IV). Moreover, ANCOVA found no significant difference for SISE between the post-test of the PL and VL groups $(F[1,65]=0.858$, $p=0.358$, partial $\eta^{2}=0.013$ ).

\section{Enjoyment}

Both the PL and VL groups improved significantly from the pre- to post-tests, $t(33)=2.20, p<0.05, d=0.35$, CI $0.95=[0.03,0.67]$ and $t(33)=2.87, p<0.01, d=$ 0.53 , CI $0.95=[0.14,0.92]$, respectively. There were small and medium effect sizes for the PL and VL (Table V). Moreover, ANCOVA found no significant difference for enjoyment between the PL and VL $(F[1,65]=0.006$, $p=0.939$, partial $\left.\eta^{2}=0.000\right)$. In sum, both the VL and PL were as effective in terms of improving students' enjoyment after the laboratory.

\section{DISCUSSION}

The aim of this study was to investigate the effectiveness of guided inquiry-based PL and VL on various learning objectives, including conceptual understanding, IP, SISE, and enjoyment. After some auxiliary factors were controlled (i.e., the curriculum, guided inquiry worksheet, time of experiment, and technology-enhanced), the conceptual test revealed that the VL was as effective as the PL for promoting simple concepts, but the VL seemed to be effective for difficult concepts. Finkelstein et al. [2] and Zacharia and de Jong [25] found that VL could promote students' performance of solving simple and complex circuits, respectively, because it affords a view of current

TABLE IV. Means, standard deviations, and comparisons of SISE.

\begin{tabular}{lccccc}
\hline \hline Group & Pretest $M(\mathrm{SD})$ & Post-test $M(\mathrm{SD})$ & $t$ & $p$ & Cohen's $d^{\text {a }}$ and its CI 0.95 \\
\hline PL $(n=34)$ & $3.29(0.55)$ & $3.46(0.48)$ & 1.96 & 0.059 & 0.33 CI $0.95=[-0.01,0.66]$ \\
VL $(n=34)$ & $2.99(0.40)$ & $3.25(0.43)$ & 2.81 & $<0.01$ & 0.62 CI $0.95=[0.15,1.10]$ \\
\hline \hline
\end{tabular}

${ }^{\mathrm{a}}$ Effect size $d=<0.3$ small effect, 0.5-0.8 medium effect, and $>0.8$ large effect.

TABLE V. Means, standard deviations, and comparisons of enjoyment.

\begin{tabular}{lccccc}
\hline \hline Group & Pretest $M(\mathrm{SD})$ & Post-test $M(\mathrm{SD})$ & $t$ & $p$ & Cohen's $d^{\text {a }}$ and Its CI 0.95 \\
\hline PL $(n=34)$ & $3.99(0.72)$ & $4.21(0.52)$ & 2.20 & $<0.05$ & 0.35 CI $0.95=[0.03,0.67]$ \\
VL $(n=34)$ & $3.82(0.63)$ & $4.13(0.53)$ & 2.87 & $<0.01$ & 0.53 CI $0.95=[0.14,0.92]$ \\
\hline \hline
\end{tabular}

${ }^{\mathrm{a}}$ Effect size $d=<0.3$ small effect, $0.5-0.8$ medium effect, and $>0.8$ large effect. 
flow. The VL showed the motion of electrons which cannot be seen in the PL. In the current study, the visualization was controlled to be the same, but the VL students still performed better on the difficult questions. Further research should be conducted to understand why this difference in understanding difficult concepts would arise. Simon [67] particularly found that students in an inquiry-based VL perceived the enhancement in analytical and critical thinking skills based on a pre- to post-survey compared with the traditional laboratories. The current study also revealed that the VL group appeared to interpret data and design new experiments better as shown by the last part of their worksheet. Both tasks belonged to higher cognitive skills. Simply put, VL seemed to have greater potential to enhance difficult conceptual understanding and high-level cognitive thinking skills.

Nevertheless, according to the responses of the worksheet, the PL seemed to promote some inquiry tasks such as setting goals for the laboratory, reviewing the pendulum motion concept, constructing experiment questions and identifying variables, planning, experimenting, and the further improvement of the experiment. This was mostly consistent with the results of Chen et al. [8] and the eye tracking system results shown by Chien et al. [30]. This result might have been influenced by students' mindsets and learning strategies [8]. Based on Chen et al.'s interviews, the VL students expressed an expectation that computer simulations were perfect; hence, they often expected them to have no manipulation restrictions or errors. Olson, Clough, and Vanderlinden [68] found an overwhelming trust in technology or computer simulation among undergraduate students. Moreover, they were amazed to undergo tremendous conditions and experiments which could not be experienced in the traditional laboratory. Therefore, the VL group tended not to conduct the experiment as systematically and carefully as the PL group did in sections V or VI. In the present study, although the VL did not yield ideal data and the worksheet asked them to improve the experiments, the VL students might have had the perception that the computers create precise data. They tended to lack a critical view of the results generated by the $\mathrm{PhET}$ pendulum. Since having a skeptical view of results is a principle of scientific inquiry [69], instructors will need to tackle this challenge if VL is widely adopted.

Furthermore, embodied cognition and cognitive loading theories may explain why PL tended to promote some inquiry tasks from the beginning section, while VL boosted the last sections such as interpreting data and designing a new experiment. Based on the patterns of how the students responded to the worksheet, PL seems to benefit more due to multiple inputs through the environment in the beginning. However, the students might have felt overloaded at the end. Chien et al. [30] observed that the PL students in their study spent most of their time working on the worksheets. They had deeper cognitive processing of the worksheet zone, while the VL students carried out more trials and paid attention to the experiment zone. The VL group tended to try out or run their experiments many more times; hence, they might have learned from doing the experiments insistently. There is a notion that $\mathrm{VL}$ is more like "doing before thinking," whereas PL is more like "thinking before doing." The open-ended questions on the worksheet used in the current study may have created a heavy loading for the students. As a result, they were so overloaded that they could not persist to the last section.

Concerning SISE, the pre- and postsurvey revealed that the VL could significantly improve it through a single intervention with medium effect size. SISE is an important learning objective and predictor. It influences how people feel, think, motivate themselves, and behave [70]. In the classroom, it drives students to persist with difficult tasks [71], and it is a strong predictor for academic achievement [72]. The improvement of SISE in the VL group might be a potential reason why they had a higher score on the difficult concepts in the post-test and the more difficult part of the worksheet (e.g., interpreting results and designing a new experiment). Finally, regarding enjoyment of inquiry, both the VL and PL groups improved significantly from the preto post-tests. To pursue enjoyable learning, either PLs or VLs are the right choice. Therefore, in particular for students who have few chances to engage in student-centered inquiry, both PLs and VLs may raise their enjoyment of inquiry.

Even Indonesian low achievers who have a strong teacher-centered culture [21] could adapt well to inquiry learning which emphasizes critical thinking, the use of metacognitive strategies, and active learning. Additionally, the students had no previous experience of guided inquiry and received no teacher help. However, they could follow the challenging activities and reported that they had more positive feelings, that is, enjoyment. Both interventions were effective in terms of promoting low achievers' enjoyment. This finding may motivate Indonesian teachers, especially to escape from the continual use of the memorizing strategy. Instead, well-designed technology-based innovative curricula should be broadly popularized. Developing countries have the same opportunities to utilize technology for enhancing learning quality.

\section{CONCLUSION AND LIMITATION}

It is essential to have an empirical base to examine the effectiveness of PLs and VLs on various learning objectives. This study provided Indonesian teachers with technology-enhanced pendulum laboratories. The empirical results could inform physics teachers about how to choose a laboratory to improve low achievement students' conceptual understanding, IP, SISE, and enjoyment. Between guided inquiry-based PL and VL, neither is utterly better than the other. VL seemed to potentially improve difficult and abstract concepts and SISE. However, PL promoted several basic inquiry skills, such as planning, 
experimenting, and the further improvement of the experiment. For the long term, the alternate use of PL and VL is recommended in the classroom since they achieve different learning objectives.

It should be noted that this study had some inherent limitations. First, the study controlled students' prior conceptual knowledge. There was no significant difference in the two groups' pretests. However, the students' prior inquiry skills were not tested so it was not possible to avoid the effect of prior inquiry skills on the guided inquiry worksheet. Second, this study may not have granted enough time for the low achievers to complete the laboratories. According to Chien et al.'s [30] eye tracking analysis, low achievers in a VL ran significantly more trials than the high achievers, who also performed significantly more trials than the high or low achievers in a PL. The VL students first tended to conduct experiments rather than focus on the worksheet. They interacted with the variables in a trial-and-error mode without reading the worksheet, while the PL students focused on the worksheet before doing the experiment. This means that the VL students did not pay as much attention to the worksheet while conducting the experiment. VL students might need more time to conduct the experiment due to trial-and-error behaviors. For low achievers, the experiment time in this study might have been insufficient. The time constraints might have influenced their learning behaviors and could be a limitation of this study.

\section{APPENDIX: WORKSHEET}

Think carefully about each step to plan your experiment. Goals setting

What learning objective (s) would you like to achieve in this laboratory? (You can choose more than 1 goal)

$\square$ Goal 1: be able to design an experiment to answer a question

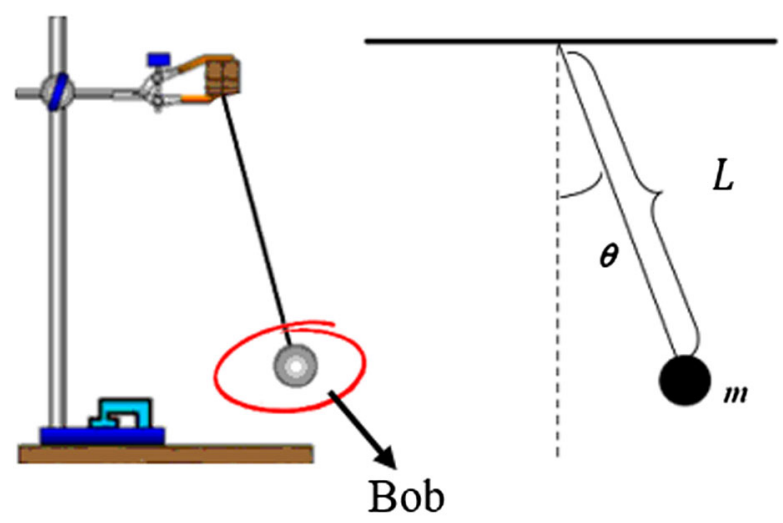

FIG. 2. Apparatus.

Goal 2: be able to describe the relationship between the period $(T)$ and the length of the pendulum rope $(L)$

Goal 3: be able to determine the gravitational acceleration of earth

Goal 4: be able to determine the control and manipulate variables in an experiment

$\square$ Goal 5: be able to figure out and solve experiment errors and change the experiment to reduce errors

Goal 6: be able to design a new experiment to investigate the relationship between the period $(T)$ and variables choosing from the mass of the bob $(m)$, the density of the bob $(\rho)$, deviation $(\theta)$, or gravitational acceleration $(g)$

$\underline{\text { The experiment materials/equipment }}$

\section{a. Physical laboratory}

(1) Stative rods (1 set) (Fig. 2)

(2) Marker (for marking the rope)

(3) Rope (at least 1,2 meter long)

(4) Bob (based on your decision)

(5) Smart tools app (downloaded from Play Store) (Fig. 3)
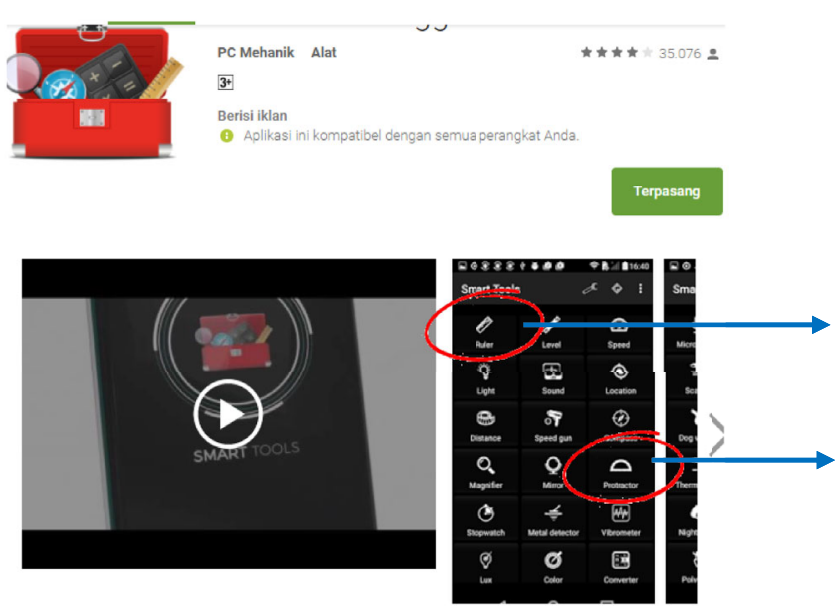

Ruler to measure the

length of the pendulum

Protractor to measure

the amplitude

FIG. 3. Smart tools app. 


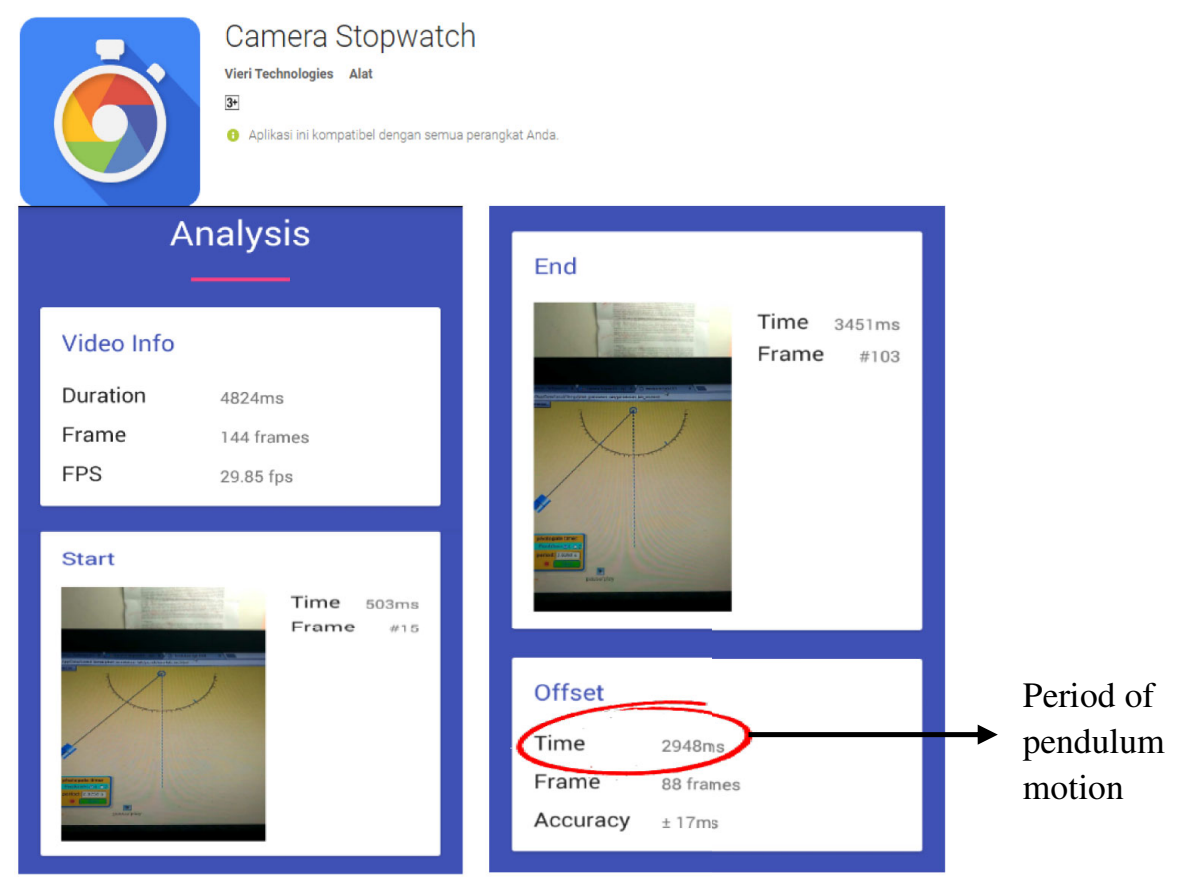

FIG. 4. Camera stopwatch app.

(6) Camera stopwatch app to measure the period (downloaded from Play Store) (Fig. 4)

\section{Guidelines to use the camera stopwatch}

(a) Record a video of your experiment "pendulum motion" by using your mobile phones

(b) Open the camera stopwatch and select the pendulum motion video from your mobile phone's storage

(c) The video will be divided into frame numbers; select the frame number (the start and the end of frame) to calculate the period

(d) Finally, you will get analysis results including video and offset information. Pay attention to the offset information (e.g., time, frame, and accuracy). The period of pendulum motion is shown as time in the offset information.

\section{b. Virtual laboratory}

- PhET interactive simulation Version 2.03.00 (Fig. 5) Review of the concept

For many years pendulums have been used for keeping time. If you pull back a pendulum and then let it go, the time it takes to swing over and then return back to its starting position is one period. Think about your experience on a swing, if you want to go faster, which means that period (T) is $\square$ smaller or $\square$ larger, the rope should be $\square$ longer or $\square$ shorter. If you swing on the moon, which means that gravity acceleration is $\square$ smaller or $\square$ larger compared with earth's gravity acceleration, it will go $\square$ faster or $\square$ slower, and the period (T) will be $\square$ smaller or $\square$ larger. Based on this information, we know that: When $T$ is larger (仓), the length of the pendulum will be $\square$ 仓 or $\square$ \} and gravity acceleration will be $\square$ 仓 or $\square$, and vice versa. Try to fill in the brackets with the relationships among these variables:

$$
\begin{aligned}
T & =\text { period } \\
L & =\text { length of pendulum, } \\
g & =\text { gravity acceleration where the pendulum works } \\
\square & =2 \pi \sqrt{\frac{\square}{\square}}
\end{aligned}
$$

However, this equation works with some restrictions. It is reasonably accurate for angles of a small degree, but for large degrees the equation will be much more complex. That is why the angular amplitude does not appear in it.

Research Question: If you want to test the relationship between $T$ and $L$, propose your research question here.

Based on your research question, determine which the control variables, independent variable and dependent variable are

- $T$ is the period of the pendulum motion which is the time the pendulum takes to swing over and then return back to its starting position (s).

- $L$ is the length of the pendulum rope (m).

- $\theta$ is the angle of deviation when you pull the pendulum.

- $m$ is the mass of the bob $(\mathrm{kg})$.

- $\rho$ is the density of the bob. If the bob is made of steel you have to see the iron's density in the density table. If you are not sure, you can calculate it based on this formula $(\rho=m / V)$. Densitiy is mass of bob/volume of bob $\left(\mathrm{kg} / \mathrm{m}^{3}\right)$ 


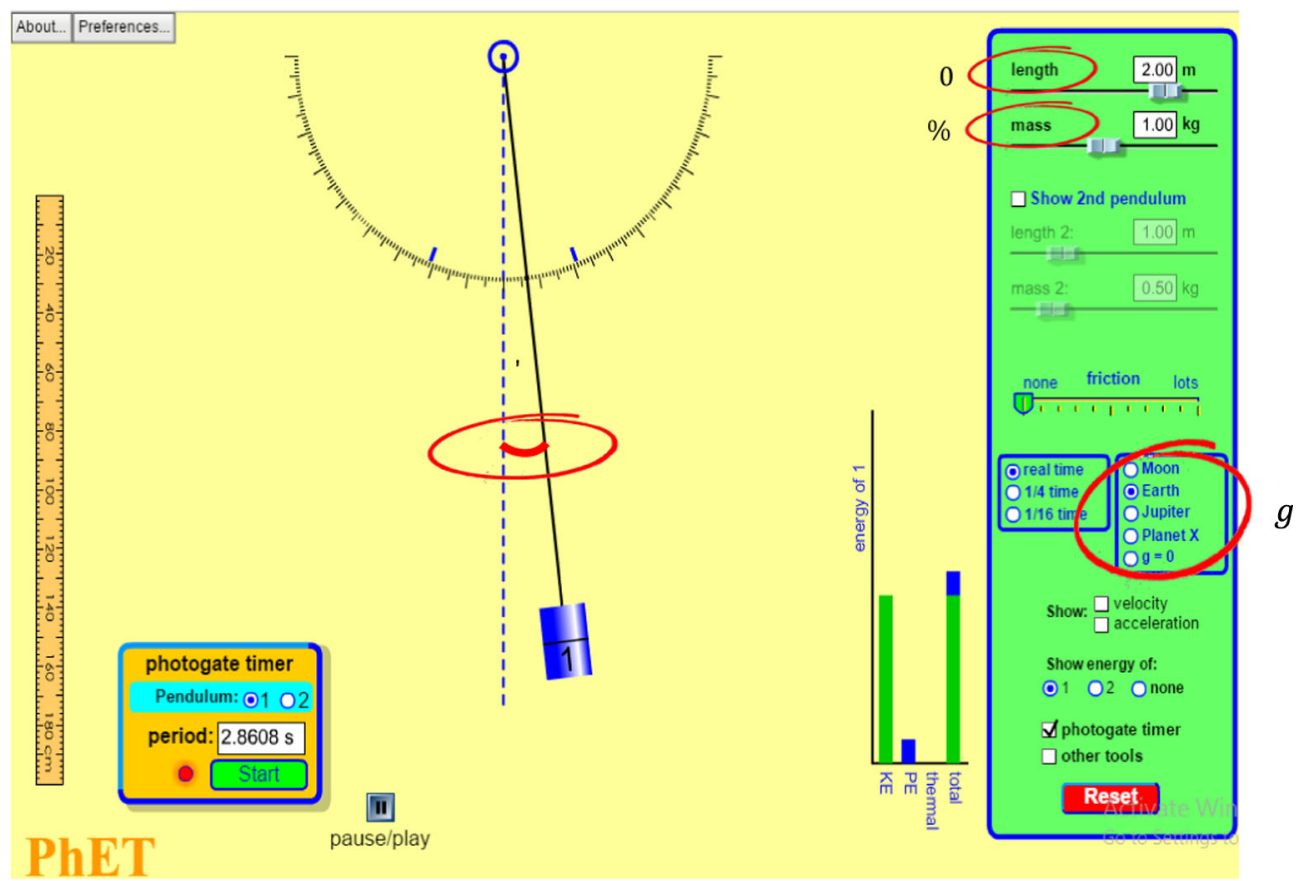

FIG. 5. Pendulum PhET.

- $g$ is gravity acceleration where the pendulum does harmonic motion $\left(\mathrm{m} / \mathrm{s}^{2}\right)$.

- Control variable

\section{$\square T \square \theta \square \rho$}

$\square L \square m \square g$

- Independent variable

$\square T \square \theta \square \rho$

$\square L \square m \square g$

- Dependent variable

$\square T \square \theta \square \rho$

$\square L \square m \square g$

Planning: Making decision about materials and the amount of data needed

(a) Think about how to manipulate the length of the pendulum and measure one period.

(b) Think about how many pairs of period and length you need to record so that you will convince others that your result is trustworthy. Why?

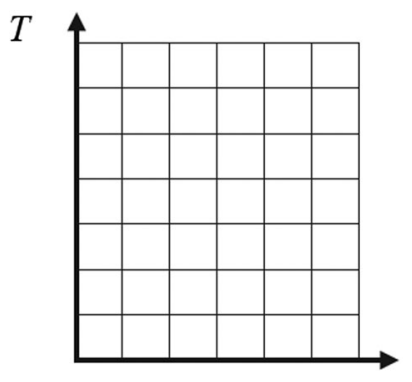

(a)

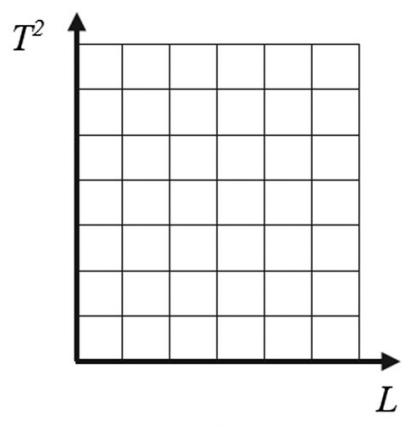

(b)
FIG. 6. (a) $L-T$ and (b) $L-T^{2}$ graphs. (c) Think about how much you give a deviation $\theta=\cdots$

(d) Predict $L-T$ and $L-T^{2}$ graphs and plot them in Fig. 6 before you start the experiment.

(e) Predict the relationship between period $(T)$ and the length of the pendulum $(L)$ in your own sentences.

Procedures

\section{a. Physical laboratory}

(1) Construct the experiment tool as shown in Fig. 2.

(2) Place it on the edge of the table.

(3) Try to use the smart tools app to measure your independent variable and use the camera stopwatch to measure your dependent variable. You can discuss it with your classmates.

(4) Swing the pendulum and report your data on the table.

\section{b. Virtual laboratory}

(1) Open PHET of pendulum motion via laptop / iPad.

(2) Set up the mass of the bob and record it on the table.

(3) Set up the length of the pendulum.

(4) Select no friction on PhET and think about how many bobs will you use in your experiment.

(5) Tick the photogate timer.

(6) Drag the bob, record the deviation angle on the table, and release it.

(7) Record the period shown by the photogate timer on the table.

Experimenting: Conducting experiments and recording data

a. Collect data, calculate the gravitational acceleration using suitable formula, and record them in Table VI. 
TABLE VI. Results for experiment I.

\begin{tabular}{|c|c|c|c|c|c|c|}
\hline No. & Pendulum length $(L)$ & Period $(T)$ & $T^{2}$ & $L / T^{2}$ & Gravitational acceleration $(g)$ & Error $|\varepsilon=(g-9.8) / 9.8|$ \\
\hline \multicolumn{7}{|l|}{1.} \\
\hline \multicolumn{7}{|l|}{2.} \\
\hline \multicolumn{7}{|l|}{3.} \\
\hline \multicolumn{7}{|l|}{4.} \\
\hline \multicolumn{7}{|l|}{5.} \\
\hline \multicolumn{7}{|l|}{6.} \\
\hline \multicolumn{7}{|l|}{7.} \\
\hline \multicolumn{7}{|l|}{8.} \\
\hline etc. & & & & & & \\
\hline
\end{tabular}

Note: Use units in International system, 4 decimals for $T, T^{2}, L / T^{2}$, and $\varepsilon$.

TABLE VII. Results for experiment II.

\begin{tabular}{|c|c|c|c|c|c|c|}
\hline No. & Pendulum length $(L)$ & Period $(T)$ & $T^{2}$ & $L / T^{2}$ & Gravitational acceleration $(g)$ & Error $|\varepsilon=(g-9.8) / 9.8|$ \\
\hline \multicolumn{7}{|c|}{1.} \\
\hline \multicolumn{7}{|l|}{2.} \\
\hline \multicolumn{7}{|l|}{3.} \\
\hline \multicolumn{7}{|l|}{4.} \\
\hline \multicolumn{7}{|l|}{5.} \\
\hline \multicolumn{7}{|l|}{6.} \\
\hline \multicolumn{7}{|l|}{7.} \\
\hline \multicolumn{7}{|l|}{8.} \\
\hline etc. & & & & & & \\
\hline
\end{tabular}

Note: Use units in International system, 4 decimals for $T, T^{2}, L / T^{2}$, and $\varepsilon$.

Evaluating: thinking about possible experimental flaws Is $g=9.8 \mathrm{~m} / \mathrm{s}^{2}$ ? Is $g$ value the same at each point? Listen to/Look at the other groups. Do they have better/ worse results? Why?

Improving experiment: Designing an experiment to improve the study

Think about how to improve the experiment to obtain $g=9.8 \mathrm{~m} / \mathrm{s}^{2} /$ decrease errors. Please use text/figures to describe your experimental design.

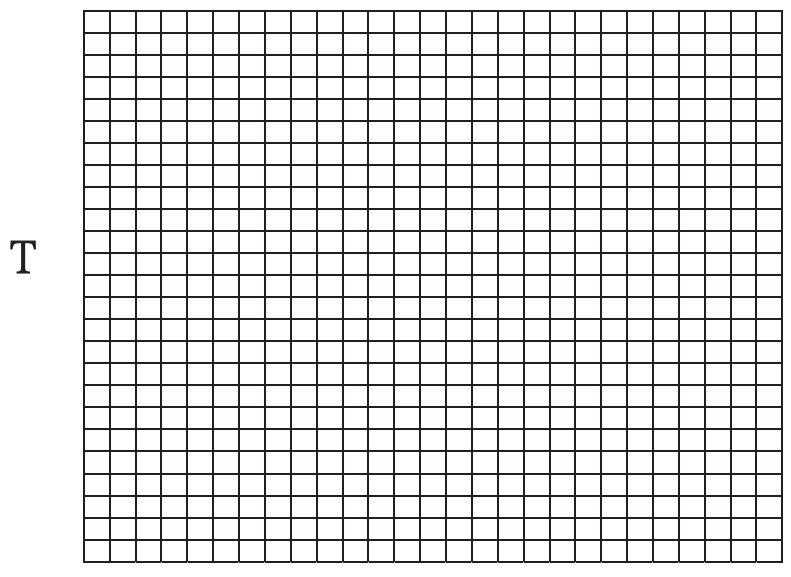

$\mathrm{L}$

FIG. 7. $L-T$ Graph.
Experimenting: Conducting own experiments

(a) Re-collect data, calculate the gravitational acceleration using suitable formula, and record them in Table VII. Control Variables: ...

(b) Analyzing errors $\left|\varepsilon_{n}=\left(\mathrm{g}_{n}-9.8\right) / 9.8\right|$ : Calculate the errors and compare with the first experiment. Do the errors $\square$ increase or $\square$ decrease? Why?

(c) Plot the $L-T$ graph in Fig. 7 .

(d) Plot the $L-T$ graph in Fig. 8.

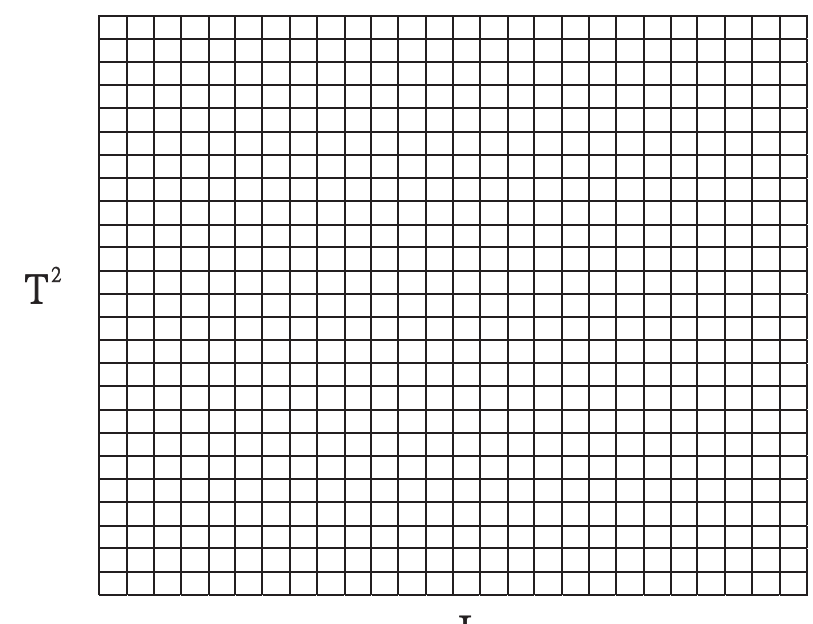

$\mathrm{L}$

FIG. 8. $\quad L-T^{2}$ graph. 
Evaluating and concluding: Evaluating results and interpreting the graphs

(a) Look at the $L-T^{2}$ graph. Is it a straight line? What do you think the slope means?

(b) Does it pass the origin (0.0) point? If not, what is the meaning of the $Y$-axis intercept?

(c) Do you think the controlled variable is well controlled? Give an explanation.
Designing a new experiment: Generating your own research questions and determining the experimental objectives

Think about variables other than the length of the pendulum which may also influence the period, for example, acceleration of gravity, amplitude, the mass of the bob, and the density of the bob. Choose the variables and design an experiment to investigate their relationship. Use text/figures to describe your ideas.
[1] K. E. Chang, Y. L. Chen, H. Y. Lin, and Y. T. Sung, Effects of learning support in simulation-based physics learning, Comput. Educ. 51, 1486 (2008).

[2] N. D. Finkelstein, W. K. Adams, C. J. Keller, P. B. Kohl, K. K. Perkins, N.S. Podolefsky, and S. Reid, When learning about the real world is better done virtually: A study of substituting computer simulations for laboratory equipment, Phys. Rev. ST Phys. Educ. Res. 1, 010103 (2005).

[3] S. Manlove, A. W. Lazonder, and T. de Jong, Collaborative versus individual use of regulative software scaffolds during scientific inquiry learning, Interact. Learn. Environ. 17, 105 (2009).

[4] T. Jaakkola, S. Nurmi, and K. Veermans, A comparison of students' conceptual understanding of electric circuits in simulation only and simulation-laboratory contexts, J. Res. Sci. Teach. 48, 71 (2011).

[5] F. Espinoza and D. Quarless, An inquiry-based contextual approach as the primary mode of learning science with microcomputer-based laboratory technology, J. Educ. Technol. Syst. 38, 407 (2010).

[6] K. Kamtoom and N. Srisawasdi, Technology-enhanced Chemistry Learning and Students' Perceptions: A Comparison of Microcomputer-based Laboratory and Webbased Inquiry Science Environment, in Proceedings of the 22nd International Conference on Computers in Education (Asia-Pacific Society for Computers in Education, Nara, Japan, 2014).

[7] A. A. Zucker, R. Tinker, C. Staudt, A. Mansfield, and S. Metcalf, Learning science in grades 3-8 using probeware and computers: Findings from the TEEMSS II Project, J. Sci. Educ. Technol. 17, 42 (2008).

[8] S. Chen, W. H. Chang, C. H. Lai, and C. Y. Tsai, A comparison of students' approaches to inquiry, conceptual learning, and attitudes in simulation-based and microcomputer-based laboratories, Sci. Edu. 98, 905 (2014).

[9] J. Dostál, Remote and virtual laboratory as a mod-ern instrument for induction of the pu-pils' inquiry activities with emphasis on the technical and science-based subjects, Proceedings of ICAICTE Annual Conference (Guangzhou, China, 2015).

[10] J. Broisin, R. Venant, and P. Vidal, Lab4CE: A remote laboratory for computer education, Int. J. Artif. Intell. Educ. 27, 154 (2017).
[11] H. Y. Wang, H. B. L. Duh, N. Li, T. J. Lin, and C. C. Tsai, An investigation of university students' collaborative inquiry learning behaviors in an augmented reality simulation and a traditional simulation, J. Sci. Educ. Technol. 23, 682 (2014).

[12] M. Umer, B. Nasir, J. A. Khan, S. Ali, and S. Ahmed, MAPILS: Mobile augmented reality plant inquiry learning system, The IEEE Global Engineering Education Conference (Athens, Greece, 2017), pp. 1443-1449.

[13] S. Chen, H. C. Lo, J. W. Lin, J. C. Liang, H. Y. Chang, and F. K. Hwang, and C. Y. Wang, Development and implications of technology in reform-based physics laboratories, Phys. Rev. ST Phys. Educ. Res. 8, 020113 (2012).

[14] R. Trumper, The physics laboratory-A historical overview and future perspectives, Sci. Educ. 12, 645 (2003).

[15] Z. C. Zacharia and C.P. Constantinou, Comparing the influence of physical and virtual manipulatives in the context of the Physics by Inquiry curriculum: The case of undergraduate students' conceptual understanding of heat and temperature, Am. J. Phys. 76, 425 (2008).

[16] K. YoungHwan and A. N. Fadli, Categorization of ICT utilization in education challenges in Indonesia based on four categories of ICT utilization challenges: Policy, infrastructure, curriculum-contents and human resources, Asia-Pac. Coll. Educ. J. 10, 31 (2014).

[17] K. Sipilä, Educational use of information and communications technology: Teachers' perspective, Technol. Pedagogy. Educ. 23, 225 (2013).

[18] K. A. Bingimlas, Barriers to the successful integration of ICT in teaching and learning environments: A review of the literature, Eurasia J. Math. Sci. Technol. Educ. 5, 235 (2009).

[19] C. Sicilia, The Challenges and benefits to teachers' practices in constructivist learning environments supported by technology, Master's thesis, McGill University, Montreal, 2006.

[20] E. Topracki, Obstacles at integration of schools into information and communication technologies by taking into consideration the opinions of the teachers and principals of primary and secondary schools in Turkey, E-J. Inst. Sci. Technol. 9, 1 (2006).

[21] K. N. Marambe, J. D. Vermunt, and H. P. A. Boshuizen, A cross-cultural comparison of student learning patterns in higher education, Higher Educ. 64, 299 (2012). 
[22] G. Olympiou and Z. C. Zacharia, Blending physical and virtual manipulatives: An effort to improve students' conceptual understanding through science laboratory experimentation, Sci. Educ. 96, 21 (2012).

[23] T. Jaakkola and S. Nurmi, Fostering elementary school students' understanding of simple electricity by combining simulation and laboratory activities, J. Comput. Assist. Learn. 24, 271 (2008).

[24] M. Başer and S. Durmuş, The Effectiveness of Computer Supported Versus Real Laboratory Inquiry Learning Environments on the Understanding of Direct Current Electricity among Pre-Service Elementary School Teachers, Eurasia J. Math. Sci. Technol. Educ. 6, 47 (2010).

[25] Z. C. Zacharia and T. de Jong, The effects on students' conceptual understanding of electric circuits of introducing virtual manipulatives within a physical manipulativesoriented curriculum, Cognit. Instr. 32, 101 (2014).

[26] M. Bajpai, Developing concepts in physics through virtual lab experiment: An effectiveness study, Techno Learn: Int. J. Educ. Technol. 3, 43 (2013).

[27] Z. C. Zacharia and G. Olympiou, Physical versus virtual manipulative experimentation in physics learning, Learn. Instr. 21, 317 (2011).

[28] Z. C. Zacharia, E. Loizou, and M. Papaevripidou, Is physicality an important aspect of learning through science experimentation among kindergarten students?, Early Childhood Res. Q. 27, 447 (2012).

[29] M. D. Renken and N. Nunez, Computer simulations and clear observations do not guarantee conceptual understanding, Learn. Instr. 23, 10 (2013).

[30] K. P. Chien, C.-Y. Tsai, H.-L. Chen, W.-H. Chang, and S. Chen, Learning differences and eye fixation patterns in virtual and physical science laboratories, Comput. Educ. 82, 191 (2015).

[31] National Research Council (NRC), Inquiry and the $\mathrm{Na}$ tional Science Education Standards: A Guide for Teaching and Learning (National Academies Press, Washington, DC, 2000).

[32] R.W. Bybee, Scientific Inquiry, and Science Teaching, in Scientific Inquiry, and Nature Science, edited by L. B. Flick and N. G. Lederman (Springer-Dordrecht, Netherlands, 2006), pp. 1-14.

[33] E. M. Furtak, T. Seidel, H. Iverson, and D. C. Briggs, Experimental and quasi-experimental studies of inquirybased science teaching: A meta-analysis, Rev. Educ. Res. 82, 300 (2012).

[34] S. Rönnebeck, S. Bernholt, and M. Ropohl, Searching for a common ground-A literature review of empirical research on scientific inquiry activities, Stud, Sci. Educ. 52, 161 (2016).

[35] L. B. Buck, S. L. Bretz, and M. H. Towns, Characterizing the level of inquiry in the undergraduate laboratory: Discrepancies abound in use of the word "inquiry". We propose a quantitative rubric to characterize inquiry in undergraduate laboratories, J. Coll. Sci. Teach. 38, 52 (2008).

[36] R. D. Purwandari, Physics laboratory investigation of vocational high school field stone and concrete construction techniques in the Central Java province (Indonesia), J. Educ. Pract. 6, 85 (2015).
[37] Dwikoranto, W. A.Surasmi, A. Suparto, S. Tresnaningsih, D. Sambada, T. Setyowati, A. Faqih, and R. Setiani, Designing laboratory activities in elementary school oriented to scientific approach for teachers SD-Kreatif Bojonegoro, J. Phys. Conf. Ser. 997, 012041 (2018).

[38] P. Bivall, S. Ainsworth, and L. A. E. Tibell, Do haptic representations help complex molecular learning?, Sci. Educ. 95, 700 (2011).

[39] G. Lakoff and M. Johnson, Philosophy in the Flesh: The Embodied Mind and its Challenge to Western Thought (Basic Books, New York, 1999).

[40] M. Wilson, Six views of embodied cognition, Psychon. Bull. Rev. 9, 625 (2002).

[41] F. Paas, A. Renkl, and J. Sweller, Cognitive load theory and instructional design: Recent developments, Educ. Psychol. 38, 1 (2003).

[42] J. Sweller, Cognitive load theory, learning difficulty, and instructional design, Learn. Instr. 4, 295 (1994).

[43] Z. C. Zacharia, Comparing and combining real and virtual experimentation: An effort to enhance students' conceptual understanding of electric circuits, J. Comput. Assist. Learn. 23, 120 (2007).

[44] V. Gallese and G. Lakoff, The brain's concepts: The role of the sensory-motor system in conceptual knowledge, Cognitive neuropsychology 22, 455 (2005).

[45] A. M. Glenberg, M. Sato, and L. Cattaneo, Use-induced motor plasticity affects the processing of abstract and concrete language, Curr. Biol. 18, R290 (2008).

[46] C. Y. Wang et al., A review of research on technologyassisted school science laboratories, J. Educ. Technol. Soc. 17, 307 (2014).

[47] S. W. Bonham, D. L. Harper, and L. Pauley, Evaluation of a multiple goal revision of a physics laboratory, AIP Conf. Proc., 82, 1513 (2013).

[48] J. R. Brinson, Learning outcome achievement in nontraditional (virtual and remote) versus traditional (handson) laboratories: A review of the empirical research, Comput. Educ. 87, 218 (2015).

[49] National Research Council (NRC), A Framework for K-12 Science Education: Practices, Crosscutting Concepts, and Core Ideas (National Academies Press, Washington, DC, 2012).

[50] A. Bandura, Social Foundations of Thought and Action: A Social Cognitive Theory (Prentice-Hall, Inc., Englewood Cliffs, NJ, 1986).

[51] A. Bandura, W. H. Freeman, and R. Lightsey, Self-efficacy: The exercise of control (WH Freeman, New York, 1999).

[52] F. Pajares, Self-efficacy beliefs in academic settings, Rev. Educ. Res. 66, 543 (1996).

[53] D. J. Ketelhut, The impact of student self-efficacy on scientific inquiry skills: An explanatory investigation in River City, a multi-user virtual environment, J. Sci. Educ. Technol. 16, 99 (2007).

[54] S. L. Britner and F. Pajares, Sources of science self-efficacy beliefs of middle school students, J. Res. Sci. Teach. 43, 485 (2006).

[55] K. Winkelmann, M. Baloga, T. Marcinkowski, C. Giannoulis, G. Anquandah, and P. Cohen, Improving students' inquiry skills and self-efficacy through research-inspired modules in 
the general chemistry laboratory, J. Chem. Educ. 92, 247 (2015).

[56] M. Rice, D. Owies, A. Campbell, R. Snow, N. Owen, and D. Holt, V-Lab: A virtual laboratory for teaching introductory concepts and methods of physical fitness and function, Australas, J. Educ. Technol. 15, 188 (1999).

[57] G. Rong, Z. Miaoliang, D. Yabo, S. Dandan, and W. Yonggu, A case study of virtual circuit laboratory for undergraduate student courses, Proceedings of the 6th International Conference on Information Technology Based Higher Education and Training (IEEE, Santo Domingo, Dominican Republic, 2005), pp. T2B/21-T2B/24.

[58] J. Michael and H. I. Modell, Active Learning in Secondary and College Science Classrooms: A Working Model for Helping the Learner to Learn (Routledge, New York, 2003).

[59] A. Raes, T. Schellens, B. De Wever, and E. Vanderhoven, Scaffolding information problem solving in web-based collaborative inquiry learning, Comput. Educ. 59, 82 (2012).

[60] D. J. Ketelhut, Assessing Gaming, Computer, and Scientific Inquiry Self-Efficacy in a Virtual Environment, In Serious Educational Game Assessment: Practical Methods, and Models for Educational Games, Simulations, and Virtual Worlds, edited by L. Annetta and S. Bronack (Sense Publisher, AW Rotterdam, Netherlands, 2011), pp. 1-18.

[61] Y. Liang, P. W. Lau, W. Y. Huang, R. Maddison, and T. Baranowski, Validity and reliability of questionnaires measuring physical activity self-efficacy, enjoyment, social support among Hong Kong Chinese children, Prev. Med. Rep. 1, 48 (2014).

[62] L. T. Hu and P. M. Bentler, Cutoff criteria for fit indexes in covariance structure analysis: Conventional criteria versus new alternatives, Struct. Equ. Modeling. 6, 1 (1999).
[63] B. G. Tabachnick and L. S. Fidell, Using Multivariate Statistics 5th ed. (Allyn \& Bacon/Pearson Education, Boston, MA, 2007).

[64] D. Iacobucci, Structural equations modeling: Fit indices, sample size, and advanced topics, J. Consum. Psychol. 20, 90 (2010).

[65] J. Algina, H. J. Keselman, and R. D. Penfield, Confidence interval coverage for Cohen's effect size statistic, Educ. Psychol. Meas. 66, 945 (2006).

[66] K. D. Bird, Analysis of Variance via Confidence Intervals (Sage, Newbury Park, CA, 2004).

[67] N. Simon, Simulated, and virtual science laboratory experiments: improving critical thinking, and higher-order learning skills, in Society for Information Technology, and Teacher Education International Conference, edited by M. Searson and M. Ochoa (Association for the Advancement of Computing in Education, Jacksonville, Florida, USA, 2014), pp. 453-459.

[68] J. K. Olson, M. P. Clough, and D. W. Vanderlinden, Undergraduates' NOS conceptions and the role of historical narratives: A very tangled web, Proceedings of the 9th International History, Philosophy of Science in Science Teaching (IHPST) Conference (Calgary, Canada, 2007), pp. 24-27.

[69] R. W. Bybee, Scientific inquiry and science teaching, Sci. Technol. Educ. Libr. 25, 1 (2006).

[70] A. Bandura, Perceived self-efficacy in cognitive development and functioning, Educ. Psychol. 28, 117 (1993).

[71] R. W. Lent, S. D. Brown, and K. C. Larkin, Relation of self-efficacy expectations to academic achievement and persistence, J. Counsel. Psychol. 31, 356 (1984).

[72] S. L. Britner and F. Pajares, Sources of science self-efficacy beliefs of middle school students, J. Res. Sci. Teach. 43, 485 (2006). 